Sepúlveda, F. y Aparicio Molina, C. (2019). Habilidades de liderazgo instruccional de directores y sus equipos de gestión de escuelas de la región del Biobío, Chile: fortalezas y desafíos.

Revista de Investigación Educativa, 37(2), 487-503.

DOI: http://dx.doi.org/10.6018/rie.37.2.329861

\title{
Habilidades de liderazgo instruccional de directores y sus equipos de gestión de escuelas de la región del Biobío, Chile: fortalezas y desafíos
}

\section{Instructional leadership skills of principals and management teams from schools of the Biobío region, Chile: Strengths and challenges}

\author{
Felipe Sepúlveda* y Carolina Aparicio Molina** \\ *Departamento Fundamentos de la Pedagogía. Facultad de Educación. Centro de Investigación en Educación y \\ Desarrollo (CIEDE). Universidad Católica de la Santísima Concepción (UCSC), Chile \\ ** Departamento Fundamentos de la Pedagogía. Facultad de Educación. Universidad Católica de la Santísima \\ Concepción (UCSC), Chile
}

\begin{abstract}
Resumen
En esta investigación se describen las habilidades de liderazgo con un enfoque instruccional de directores, equipo de gestión y docentes de establecimientos educativos de la región del Biobío, Chile. Se encuestaron a 64 miembros del equipo de gestión y 424 docentes de 17 establecimientos educativos utilizando el instrumento PIMRS. El análisis de datos demostró que no existen diferencias significativas en la valoración de los encuestados en la implementación de las tres dimensiones establecidas en el PIMRS. Sin embargo, se determinó que los docentes presentan diferencias significativas con respecto a los miembros del equipo directivo en término de las tres dimensiones y diez funciones establecidas en el PIMRS. Al comparar la valoración de los equipos directivos y los docentes, las menores brechas se registran en las funciones asociadas a la definición de la misión de la escuela y las mayores brechas se encuentran en las funciones asociadas al fomento de un adecuado clima de aprendizaje escolar. La valoración de las activi-
\end{abstract}

Correspondencia: Felipe Sepulveda, fsepulveda@ucsc.cl, Universidad Católica de la Santísima Concepción, Facultad de Educación, Departamento Fundamentos de la Pedagogía, Av. Alonso de Ribera 2850, Concepción, Región del Bío Bío, Chile 
dades asociadas a la implementación de liderazgo instruccional de los directores y jefes UTP difiere en términos de los énfasis que se otorgan dependiendo de cada cargo.

Palabras clave: liderazgo instruccional; liderazgo; administración escolar; directores.

\begin{abstract}
This research describes leadership skills from an instructional approach of school principals, management team and teachers of educational organizations from the Biobio region, Chile. A sample of 64 members of the school management team and 424 teachers were surveyed using the PIMRS instrument. The data analysis showed no significant differences in the assessment of the respondents in the implementation of the three dimensions established in the PIMRS. However, it was determined that teachers have significant differences with respect to the members of the management team in terms of the three dimensions and ten functions established in the PIMRS. When comparing the assessment of the management teams and the teachers, the smallest gaps are found in the functions associated with the definition of the school's mission and the biggest gaps are found in the functions associated with the promotion of an adequate school learning climate. The evaluation of the activities associated with the implementation of instructional leadership of principals and pedagogical heads differs in terms of the emphasis that is granted depending on each position.

Keywords: Instructional leadership; Leadership; School, Administration; Principals.
\end{abstract}

\title{
Introducción
}

La literatura especializada coincide en que el rol que desempeña el director del establecimiento es un componente relevante sobre los procesos de enseñanza y aprendizaje que ocurren al interior de los centros educativos. El meta-análisis de los resultados reportados en una gran cantidad de estudios con respecto a la incidencia de los directores de escuelas sobre el desempeño escolar confirman sistemáticamente esta relación (Leithwood, Day, Sammons, Harris \& Hopkins, 2006; Marzano, Waters \& McNulty, 2005; Robinson, Lloyd \& Rowe, 2008). Como queda establecido en estas investigaciones, la efectividad de los directores escolares depende de los enfoques de liderazgo empleado y la consistencia en el desempeño directivo que facilite establecer procesos de cambio educativo. Así mismo, los centros educativos que logran altos estándares de logros académicos no son el resultado del trabajo aislado de un director de excelencia. Un líder efectivo se caracteriza por crear un clima donde los miembros del equipo logran transformar desafíos complejos en resultados excepcionales (Kouzes \& Posner, 2012). En la actualidad se espera que los directores de escuela se transformen en agentes que faciliten procesos de reflexión sostenida sobre las prácticas pedagógicas que acontecen en sus centros educativos. Fullan (2014), utiliza el concepto de liderar el aprendizaje para referirse al rol del director para liderar a los docentes de sus escuelas en un proceso de aprendizaje para mejorar la enseñanza, en el proceso se logra comprender de mejor manera qué estrategias son efectivas y cuáles no lo son. Esta mirada de liderazgo escolar requiere promover el aprendizaje de los docentes conformando equipos colaborativos, donde el director de la escuela asume el rol de facilitador en la creación de una cultura que promueve el aprendizaje sostenido (Dufour \& Marzano, 2009). Siguiendo esta tendencia hacia el fortalecimiento de las 
competencias profesionales del equipo docente Hargreaves y Fullan (2014), promueven la importancia de fomentar el capital profesional al interior de cada escuela, referido a "la definición de elementos críticos con respecto a lo que se requiere para crear una elevada calidad y rendimiento en el ejercicio de todas las profesiones, incluyendo la enseñanza" (p. 132). Actividad clave del desempeño directivo en la actualidad.

\section{Contexto regulatorio sobre liderazgo escolar en Chile}

Las crecientes demandas hacia el mejoramiento educativo producto de un sistema globalizado, que exige altas capacidades técnicas para lograr ser competitivo, se ve reflejado en la importancia que atribuyen los estados a mediciones internacionales tales como PISA, TIMSS, PIRLS, TERCE, ICCS, entre otras, que son asociadas a los niveles de logros educativos de cada país participante. En América Latina esto ha incentivado el desarrollo de políticas y reformas educativas dirigidas hacia mejorar los estándares de calidad escolar de cada país. Respecto a liderazgo escolar, existe un amplio campo para la mejora, como establece el informe de la Oficina Regional de Educación para América Latina y el Caribe de la Organización de las Naciones Unidas para la Educación, la ciencia y la Cultura (OREALC-UNESCO, 2015) "existen iniciativas recientes a favor del rol directivo, sin embargo subsisten dificultades de coherencia interna entre las acciones en curso y carencias en ámbitos decisivos (por ejemplo, la formación profesional) para lograr un liderazgo directivo eficaz en las escuelas"' (p. 18). La revisión de las políticas que definen el rol de los directores escolares en América Latina indica que existe un excesivo centralismo administrativo, en deterioro de la independencia de los directores de cada establecimiento para liderar procesos de mejora escolar (Flessa, Bramwell, Fernández \& Weinstein, 2018) Así mismo, existen desafíos con respecto a las competencias asociadas al liderazgo escolar que presentan los actuales directores de establecimientos educativos. Como ejemplo, un estudio que analizó las prácticas de liderazgo más utilizadas en 8 sistemas educativos sobre la base de mediciones PISA 2012, encontró que la toma de decisiones basada en evidencias no es una práctica común en los directores de América Latina (Vaillant \& Zidán, 2016).

Siguiendo con la tendencia internacional, las reformas educativas impulsadas en Chile recogen la relevancia del liderazgo escolar apuntando a fortalecer el rol de los directivos. Al igual que en otros sistemas educativos, los directores de escuelas tienden a centrar una significativa parte de su tiempo a labores administrativas y no en tareas relacionadas con liderar procesos de mejora pedagógica. Murillo (2012), indica que los directores de escuela en Chile son los que utilizan más tiempo en funciones administrativas (31.3\% de su tiempo), dejando otro tercio de su tiempo (31.1\%) a labores instruccionales como el monitoreo de la enseñanza, evaluaciones, orientación de los profesores e implementación de currículo (Murillo, 2012). Atendiendo a estos desafíos, últimamente se han propulsado diferentes políticas dirigidas al fortalecimiento del rol de los equipos directivos del sistema escolar chileno. Por un lado, se han propuesto iniciativas legales dirigidas a definir las responsabilidades y atribuciones asociadas a los cargos directivos. Dentro de esta línea es posible mencionar la Ley de Calidad y Equidad de la Educación del Ministerio de Educación de Chile (en adelante, Mineduc), que establece la posibilidad de evaluar el desempeño de los directores y ofrece cierta autonomía para que el director 
pueda proponer a su equipo de gestión (Mineduc, 2011a). Adicionalmente la ley que crea un nuevo Sistema de Educación Pública promueve entregar mayor autonomía a cada establecimiento educativo en términos de la administración de recursos, planificación estratégica y selección de perfiles profesionales (Mineduc, 2017). Otros avances apuntan a mejorar las capacidades de liderazgo asegurando la selección de los profesionales del área de la educación más idóneos para ocupar cargos de gestión y promoviendo formación continua de los directores actualmente en ejercicio. Dentro de estas iniciativas destaca la promulgación del Marco para la Buena Dirección y el Liderazgo Escolar (Mineduc, 2015) que define las prácticas, competencias y conocimientos para el desarrollo del liderazgo escolar en el país. Adicionalmente durante los últimos años se ha implementado el Plan de Formación de Directores que financia la preparación avanzada de directores en programas de postgrado con calidad acreditada. Todo lo anterior en un proceso de reforma educativa de largo aliento que pretende impactar en la calidad y equidad de la educación chilena.

\section{Dirección escolar a través del enfoque de liderazgo instruccional}

La reciente reforma educativa implementada en Chile, tiende a poner el énfasis en los procesos de enseñanza y el aprendizaje que ocurren en el aula. Así, la implementación del Sistema de Aseguramiento de la Calidad (Mineduc, 2011b) tiene como objetivo central asegurar el acceso a una educación de calidad con equidad para todos los estudiantes del país implementado procesos de evaluación integral de cada establecimiento educativo reconocido por el Ministerio de Educación de Chile. En consecuencia, los equipos de gestión escolar deben ser capaces de diseñar e implementar un plan de mejoramiento educativo que asegure el cumplimiento de objetivos a corto plazo (1 año) y mediano plazo (4 años). Es en este contexto, que el liderazgo instruccional entendido como las acciones que vinculan el liderazgo con el aprendizaje, toma más relevancia. En la actualidad el liderazgo instruccional también es entendido bajo los conceptos liderazgo pedagógico, el liderazgo curricular y el liderazgo para el aprendizaje (Bush, 2016).

El concepto de liderazgo instruccional no es nuevo en el campo de la educación. Esta aproximación destaca como uno de los referentes más importantes asociado al liderazgo directivo pedagógico y curricular (Maureira, 2018; Sepúlveda \& Aparicio, 2017). Durante los años 80s este enfoque de liderazgo se restringía a la definición de una concepción de funciones muy bien establecidas para los directores de escuela, visión que ha avanzado hacia una aproximación del liderazgo escolar basada en resultados de investigaciones empíricas y sustentada en la teoría (Hallinger \& Wang, 2015).

Un estudio de revisiones de la literatura relacionada al liderazgo en educación reconoce que una aproximación de liderazgo instruccional presenta evidencia empíricamente verificada en el desempeño académico de los estudiantes, en comparación a otros enfoques de liderazgo escolar (Hallinger, 2011; Robinson et al., 2008). Con el propósito de evaluar las conductas asociadas al desarrollo de una aproximación de liderazgo de tipo instruccional Hallinger \& Murphy (1985), diseñaron la escala de apreciación de la gestión instruccional de directores (PIMRS por sus iniciales en inglés). Este instrumento ha sido ampliamente utilizado para el desarrollo de estudios empíricos por más de 200 investigadores en diferentes sistemas escolares de 26 países (Hallinger, 2011). Sin embargo, la gran mayoría de estos estudios han sido desarrollados en países Anglo-Parlantes y de Asia. 
En consideración a la reforma educativa chilena que enfatiza los procesos de aseguramiento de la calidad, es que el accionar de equipos directivos toma relevancia en su implementación. Si bien, en Chile se han desarrollado esfuerzos para describir y sistematizar el accionar de directores de las escuelas (ver Weinstein \& Muñoz, 2012, para una revisión), aún es necesario avanzar para comprender cuáles modelos de liderazgo se aproximan más al desempeño de los directores. Adicionalmente, en el contexto chileno también es necesario poner atención al desempeño de los equipos de gestión escolar para comprender mejor quienes son los referentes pedagógicos de la escuela (Flessa et al., 2018). Así en esta investigación se analiza la percepción de los docentes con respecto a la implementación de acciones asociadas al desarrollo de un enfoque de liderazgo instruccional por parte de todo el equipo directivo de la escuela. Junto a lo descrito se registró la autopercepción en la ejecución de actividades de liderazgo instruccional de los miembros de los equipos de gestión escolar para poder describir los énfasis en el despliegue de actividades asociadas a este enfoque de liderazgo de acuerdo a la posición ocupada en el establecimiento educativo.

\section{Método}

\section{Objetivos}

En esta investigación se utiliza el enfoque de liderazgo instruccional para comprender las prácticas de liderazgo utilizadas por los equipos de gestión en establecimientos educacionales chilenos. El objetivo de la investigación se orienta a describir las fortalezas y desafíos con respecto a las habilidades de liderazgo instruccional de directores y a sus equipos de gestión de instituciones educacionales ubicadas en la región de Biobío, Chile.

\section{Población y muestra}

El estudio fue desarrollado en establecimientos educativos localizados en 12 comunas de la región del Biobío, Chile. En esta región se encuentra un total de 1556 centros educativos de nivel de parvulario, básica y media. Es la segunda región de Chile en términos de la cantidad de centros educativos reconocidos por el estado, empleando a un total de 30.861 docentes en todos sus niveles. En esta región el 56\% de los establecimientos presentan una administración municipal (financiamiento público) y el $41 \%$ son establecimientos con una administración particular-subvencionada (financiamiento compartido entre el estado y los apoderados de cada establecimiento). Finalmente, el $3 \%$ de los establecimientos son privados y no reciben ningún aporte del estado en su financiamiento.

Un total de 17 establecimientos participaron en la toma de muestras; 13 de ellos forman parte del sistema de colegios públicos (administración municipal) y 4 son colegios particulares subvencionados (financiamiento compartido entre el estado y apoderados del colegio). En total fueron encuestados 488 profesionales de la educación dentro de los que se incluyen al Director del establecimiento, Jefes de UTP (Unidad Técnico-Pedagógica), miembros del equipo de gestión del establecimiento (subdirectores, coordinadores de 
ciclo, curriculistas, orientadores e inspectores) y docentes de aula (tabla 1). Conforme a la muestra, se observa que la moda informada para los años de experiencia de los directores es de 10-15 años en el cargo. Sin embargo, los docentes que cumplen otras funciones de liderazgo dentro del colegio (Jefes UTP y miembros del equipo de coordinación) tienden a presentar menos de 10 años experiencia en esas funciones. En el caso de los docentes de aula, se observa que predomina claramente los grupos que informaron tener ente 5-9 (31\%) y más de 15 años de experiencia (29\%).

Tabla 1

Participantes del estudio caracterizados por sus funciones y años de experiencia

\begin{tabular}{lccccccc}
\hline Cargos & $\mathbf{1}$ año & $\begin{array}{c}\mathbf{2 - 4} \\
\text { años }\end{array}$ & $\begin{array}{c}\mathbf{5 - 9} \\
\text { años }\end{array}$ & $\begin{array}{c}\mathbf{1 0 - 1 5} \\
\text { años }\end{array}$ & $\begin{array}{c}\text { Más de } \\
\mathbf{1 5} \text { años }\end{array}$ & No responde & Total \\
\hline Director & 2 & 1 & 2 & 5 & 2 & 1 & 13 \\
Jefe UTP & 3 & 4 & 4 & 0 & 5 & 6 & 22 \\
Coordinador & 7 & 5 & 7 & 0 & 4 & 6 & 29 \\
Docentes & 22 & 82 & 133 & 50 & 122 & 15 & 424 \\
\hline Total & 34 & 92 & 146 & 55 & 133 & 28 & 488 \\
\hline
\end{tabular}

\section{Instrumento}

Las habilidades de liderazgo instruccional de los directores y sus equipos directivos fueron descritas utilizando la "Escala de Percepción de la Gestión Instruccional de Directores" (Principal Instructional Management Rating Scale, PIMRS) en su versión traducida al español (Fromm, Hallinger, Volante \& Wang, 2017). Este instrumento se encuentra basado en un modelo conceptual que propone tres dimensiones ordenadas en 10 funciones directivas para el apropiado desarrollo de un enfoque de liderazgo instruccional (tabla 2).

La primera dimensión concierne al rol del director para trabajar junto con el cuerpo docente y asegurar que la escuela genere una misión clara y coherente con el progreso académico de los estudiantes del establecimiento educacional. La segunda dimensión compromete al trabajo del director en coordinar y controlar el plan académico del colegio, considerando las capacidades del director de delegar y compartir la responsabilidad con los especialistas de cada área académica del establecimiento. Finalmente la tercera dimensión se refiere actividades asociadas a crear una cultura escolar que favorezca la excelencia basada en el rigor académico y altos estándares de desempeño profesional. Las funciones incluidas en la tercera dimensión se centran en que el director logre el desarrollo de una cultura que privilegia y recompensa el desarrollo de nuevas capacidades profesionales y el aprendizaje continuo. 
Tabla 2

Modelo conceptual de liderazgo instruccional de P. Hallinger

\begin{tabular}{lll}
\hline Dimensiones & \multicolumn{1}{c}{ Funciones directivas } \\
\hline Define la misión de la escuela & - & Establece las metas de la escuela \\
& - & Comunica las metas de la escuela \\
Administra el plan académico & - & Gestiona el currículo académico \\
de la escuela & - & Supervisa y evalúa la instrucción \\
Fomenta un adecuado clima & - & Monitorea el progreso académico de los estudiantes \\
de aprendizaje escolar & - & Protege el tiempo de instrucción \\
& - & Provee incentivos para los profesores \\
& - & Provee incentivos para el aprendizaje \\
& - & Promueve el desarrollo profesional \\
& - & Mantiene una alta visibilidad \\
\hline
\end{tabular}

Fuente. Adaptado de Sepúlveda \& Aparicio, 2017

Para cada una de las 10 funciones establecidas para el desarrollo de las tres dimensiones de liderazgo instruccional, el PIMRS presenta 5 preguntas dirigidas a evaluar la frecuencia de ocurrencia de los comportamientos específicos indicados en cada afirmación (50 afirmaciones en total). Cada pregunta esta rankeada con una escala de tipo Likert que varía de (1) casi nunca a (5) casi siempre. El estudio original de validación del instrumento encontró que el PIMRS cumple con altos estándares de confiabilidad, donde cada una de la 10 sub escalas evaluadas excedieron .80 en el test de consistencia interna de Cronbach (Hallinger, 2012). Análisis posteriores con respecto a la confiabilidad del instrumento basados en el meta- análisis de una serie de estudios que utilizaron el PIMRS en diferentes: niveles del sistema escolar, culturas y países (por ejemplo Estados Unidos y Asia), mostraron índices de confiabilidad del instrumento en cada una de las tres dimensiones medidas por sobre 0.88 (Hallinger, Wang \& Chen, 2013).

\section{Procedimiento de recogida y análisis de datos}

La toma de datos se concertó a través de una reunión con los directores de cada establecimiento para explicar el objetivo general de la investigación y acordar el momento más adecuado para recoger los datos del equipo de gestión y equipo docente de cada establecimiento. Según lo acordado con cada director se acudió al centro educativo durante jornadas de reunión de profesores usualmente utilizadas para planificación de asuntos internos de cada colegio y coordinación entre equipos docentes. En esta reunión, un miembro del equipo de investigación explicó el propósito de la actividad indicando que la participación era de carácter voluntario y que se mantendría el anonimato de 
las respuestas individuales de cada participante. Se les solicitó a los docentes de cada establecimiento evaluar el grado de implementación de las 50 afirmaciones establecidas en el PIMRS considerando la actuación de todo el equipo directivo del establecimiento. Por otro lado, se le solicitó a cada miembro del equipo directivo contestar las 50 afirmaciones establecidas en el PIMRS considerando las funciones que cada uno desempeña desde su cargo directivo. De esta manera, en este estudio, los resultados representan la implementación de actividades asociadas al liderazgo instruccional por parte de todo el equipo directivo. Por otra parte, la sistematización de los datos considerando el cargo directivo busca conocer el tipo de actividades más o menos desarrolladas, considerando la función en el establecimiento. Finalmente, las respuestas de los docentes indican la percepción de los mismos, con respecto al desarrollo de las funciones asociadas al liderazgo instruccional por parte del equipo directivo del establecimiento escolar en pleno.

\section{Resultados}

El propósito central de la investigación es describir el grado de desarrollo de habilidades de liderazgo instruccional por parte de los equipos directivos de establecimientos educativos de la región del Biobío, Chile. Se utilizó el modelo conceptual propuesto por Hallinger (2012) que considera tres dimensiones y diez funciones de aplicación de este enfoque de liderazgo escolar, medido utilizando una escala de valoración de 50 afirmaciones (PIMRS). A continuación, se presentan los resultados encontrados en este estudio, considerando la consistencia interna del instrumento utilizado y los valores promedio de las respuestas al instrumento por parte de todos los participantes en el estudio.

\section{Consistencia interna del instrumento}

Se verificó la consistencia interna del PIMRS de acuerdo a las respuestas de los participantes y el cargo que ocupaban en sus establecimientos educativos. Así, los valores de a de Cronbach para el los 50 del PIMRS fueron de .975 para los Directores, .935 para los Jefes UTP, .943 para los coordinadores y .977 para los docentes que respondieron el instrumento. En todos los casos se observa una alta consistencia interna del instrumento. De acuerdo a lo propuesto por Field (2013), para los casos en que un instrumento se encuentre conformado por diferentes constructos, es recomendable el cálculo de a de Cronbach para cada uno de ellos de manera separada. De esta manera, la consistencia interna por Dimensión (tabla 3) y por Función Directiva (tabla 4) son presentadas de acuerdo al cargo ocupado en los centros educativos.

Los resultados en el cálculo de a de Cronbach detallando la Dimensión y Funciones Directivas, considerando los cargos de los participantes sustentan una alta consistencia interna de instrumento utilizado. Para el caso de las Dimensiones los valores de a de Cronbach variantes entre .783 a .959. En el caso de las Funciones Directivas los valores de a de Cronbach variantes entre .654 a .944 . Al observar las tablas 3 y 4 se puede observar que los valores de a más bajos consistentemente se presentan para los Jefes UTP considerando las Dimensiones y Funciones Directivas definidas en el instrumento de medición. Posiblemente las funciones establecidas para este cargo varían mucho dependiendo el establecimiento educativo, lo que podría ser una explicación para 
Tabla 3

Valores de a de Cronbach para cada Dimensión del PIMRS y cargo en establecimiento educativo

\begin{tabular}{lcccc}
\hline Dimensiones & Director & Jefe UTP & $\begin{array}{c}\text { Equipo } \\
\text { de } \\
\text { Gestión }\end{array}$ & Docentes \\
\hline $\begin{array}{l}\text { Define la misión de la escuela } \\
\begin{array}{l}\text { Administra el plan académico de la } \\
\text { escuela }\end{array}\end{array}$ & .889 & .783 & .878 & .915 \\
$\begin{array}{l}\text { Fomenta un adecuado clima de } \\
\text { aprendizaje escolar }\end{array}$ & .939 & .894 & .886 & .951 \\
\hline
\end{tabular}

Tabla 4

Valores de a de Cronbach para cada Función Directiva del PIMRS y cargo en establecimiento educativo

\begin{tabular}{|c|c|c|c|c|}
\hline Funciones & Director & Jefe UTP & $\begin{array}{l}\text { Equipo } \\
\text { de } \\
\text { Gestión }\end{array}$ & $\begin{array}{l}\text { Docen- } \\
\text { tes }\end{array}$ \\
\hline Establece las metas de la escuela & .817 & .654 & .884 & .896 \\
\hline Comunica las metas de la escuela & .808 & .755 & .792 & .843 \\
\hline Gestiona el currículo académico & .877 & .833 & .878 & .891 \\
\hline Supervisa y evalúa la instrucción & .877 & .681 & .869 & .905 \\
\hline $\begin{array}{l}\text { Monitorea el progreso académico de la } \\
\text { instrucción }\end{array}$ & .816 & .823 & .722 & .912 \\
\hline Protege el tiempo de instrucción & .897 & .720 & .821 & .866 \\
\hline Provee incentivos para los profesores & .869 & .740 & .811 & .851 \\
\hline Provee incentivos para el aprendizaje & .917 & .737 & .860 & .928 \\
\hline Promueve el desarrollo profesional & .856 & .657 & .850 & .910 \\
\hline Mantiene una alta visibilidad & .944 & .718 & .845 & .893 \\
\hline
\end{tabular}


la alta variabilidad en las respuestas a las actividades relacionadas con el liderazgo instruccional incluidas en el instrumento utilizado.

\section{Habilidades de liderazgo instruccional de equipos de gestión}

La autopercepción de los integrantes de equipos de gestión escolar y la percepción de los docentes con respecto a la valoración en la ejecución de actividades asociadas a liderazgo instruccional fue registrada considerando las tres dimensiones y diez funciones propuestas en el instrumento utilizado en el estudio.

El análisis descriptivo de los datos recogidos y ordenados de acuerdo al cargo ocupado en el centro educativo indica diferencias en la percepción entre los integrantes del equipo de gestión y docentes considerando la valoración promedio de todos los ítems que conforman cada dimensión del modelo de aplicación de un enfoque de liderazgo instruccional (tabla 5). Las mayores brechas se observan entre la autopercepción de los Jefes UTP con los docentes de aula para las tres dimensiones del modelo.

Tabla 5

Estadísticos descriptivos de la valoración del desarrollo de actividades asociadas a las tres Dimensiones comprendidas para la ejecución de un enfoque de liderazgo instruccional considerando el cargo ocupado en el centro escolar. Se informa la media y desviación típica de todos los ítems considerados para cada dimensión

\begin{tabular}{lcccc}
\hline Dimensiones & Director & Jefe UTP & $\begin{array}{c}\text { Equipo de } \\
\text { Gestión }\end{array}$ & Docentes \\
\hline Define la misión de la escuela & $3.9 \pm .71$ & $4.1 \pm .51$ & $4.0 \pm .67$ & $3.6 \pm .84$ \\
$\begin{array}{l}\text { Administra el plan académico } \\
\text { de la escuela }\end{array}$ & $3.6 \pm .86$ & $4.0 \pm .67$ & $3.7 \pm .72$ & $3.3 \pm .95$ \\
$\begin{array}{l}\text { Fomenta un adecuado clima de } \\
\text { aprendizaje escolar }\end{array}$ & $3.9 \pm .75$ & $4.1 \pm .40$ & $3.9 \pm .62$ & $3.1 \pm .90$ \\
\hline
\end{tabular}

Se realizó un análisis de varianza 4×3 (Cargo x Dimensión) para evaluar diferencias entre la percepción en la implementación de las actividades asociadas al desarrollo de liderazgo instruccional considerando el cargo en el establecimiento y las tres dimensiones definidas por el PIMRS. A través del análisis se determinó que existen diferencias significativas en la percepción en la implementación de actividades asociadas al liderazgo instruccional considerando los cargos que se desempeñan en el establecimiento educativo, $F(3,1442)=27.147, p<.001, \eta_{\mathrm{p}}{ }^{2}=.053$. Un análisis post hoc, de tipo Bonferroni reveló que no existen diferencias en la implementación de actividades de liderazgo instruccional considerando la autopercepción de los miembros de equipo directivo (Director, Jefe UTP y Coordinadores, $p>$.05), sin embargo, la percepción de los docentes con respecto al desarrollo de actividades asociadas al liderazgo instruccional por parte del equipo directivo fue significativamente menor, $p=.006$. No 
se detectaron diferencias significativas al comparar el desarrollo de actividades de liderazgo instruccional entre las tres dimensiones comprendidas por el PIMRS, F (2, $1442)=2.319, p=.099, \eta_{\mathrm{p}}{ }^{2}=.003$, ni una interacción significativa entre el cargo y las dimensiones del instrumento sobre la base del desarrollo de actividades de liderazgo instruccional $F\left(6,1442=1.350, p=.232, \eta_{\mathrm{p}}{ }^{2}=.006\right)$.

El análisis de la valoración promedio sobre la base de las diez funciones del modelo de liderazgo instruccional ordenados de acuerdo al cargo ocupado en el centro educativo se presentan en la tabla 6. En términos generales se observa que existe una tendencia a que los profesionales que ocupan cargos de gestión presenten una valoración mayor a los docentes de aula con respecto a la ejecución de actividades asociadas al liderazgo instruccional. Por parte de los docentes, se registra una percepción de que las funciones menos presentes por parte del equipo directivo son las de proveer incentivos para los profesores $(M=2.9)$ y proveer incentivos para el aprendizaje $(M=2.7)$. Ambas funciones forman parte de la tercera dimensión del modelo de liderazgo instruccional referido a fomentar un clima adecuado para el aprendizaje escolar.

Tabla 6

Estadísticos descriptivos de la valoración del desarrollo de actividades asociadas a las diez Funciones Directivas comprendidas para la ejecución de un enfoque de liderazgo instruccional considerando el cargo ocupado en el centro escolar. Se informa la media y desviación típica de todos los ítems considerados para cada Función Directiva.

\begin{tabular}{|c|c|c|c|c|}
\hline Funciones & Director & Jefe UTP & $\begin{array}{c}\text { Equipo de } \\
\text { Gestión }\end{array}$ & Docentes \\
\hline Establece las metas de la escuela & $4.0 \pm .80$ & $4.2 \pm .51$ & $4.1 \pm .71$ & $3.6 \pm .91$ \\
\hline Comunica las metas de la escuela & $3.9 \pm .72$ & $4.0 \pm .67$ & $3.9 \pm .73$ & $3.5 \pm .90$ \\
\hline Gestiona el currículo académico & $3.4 \pm .99$ & $4.0 \pm .85$ & $3.6 \pm .92$ & $3.3 \pm 1.02$ \\
\hline Supervisa y evalúa la instrucción & $3.9 \pm .83$ & $4.2 \pm .58$ & $3.8 \pm .78$ & $3.5 \pm 1.00$ \\
\hline $\begin{array}{l}\text { Monitorea el progreso académico de } \\
\text { la instrucción }\end{array}$ & $3.3 \pm .90$ & $3.9 \pm .79$ & $3.7 \pm .75$ & $3.1 \pm 1.10$ \\
\hline Protege el tiempo de instrucción & $3.8 \pm .83$ & $4.2 \pm .58$ & $3.7 \pm .76$ & $3.1 \pm 0.99$ \\
\hline Provee incentivos para los profesores & $3.7 \pm .91$ & $3.9 \pm .79$ & $4.1 \pm .78$ & $2.9 \pm 1.03$ \\
\hline Provee incentivos para el aprendizaje & $3.9 \pm .96$ & $4.0 \pm .62$ & $3.7 \pm .88$ & $2.7 \pm 1.21$ \\
\hline Promueve el desarrollo profesional & $3.9 \pm .77$ & $4.2 \pm .46$ & $3.9 \pm .78$ & $3.3 \pm 1.08$ \\
\hline Mantiene una alta visibilidad & $4.1 \pm .96$ & $4.2 \pm .53$ & $4.1 \pm .74$ & $3.4 \pm .99$ \\
\hline
\end{tabular}




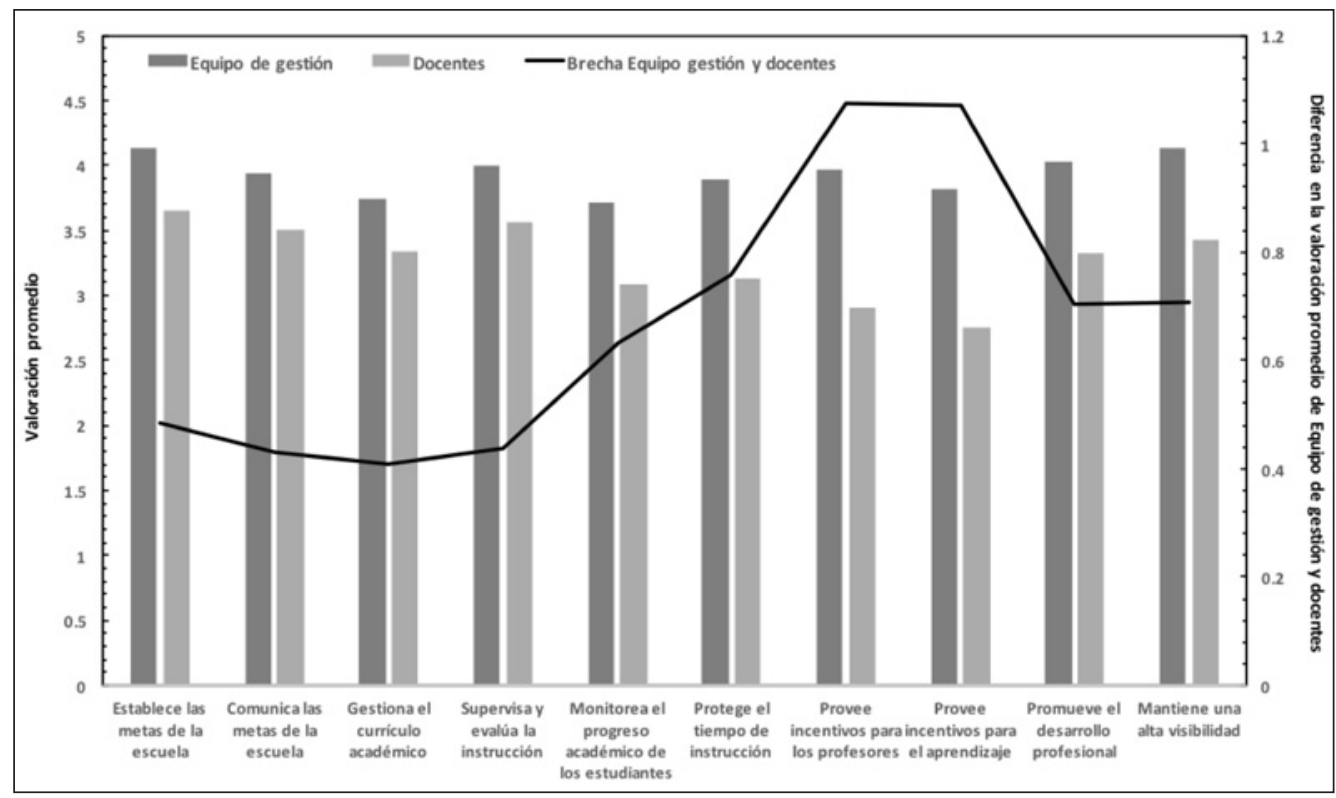

Figura 1. Valoración promedio de los miembros del equipo de gestión y docentes con respecto a las funciones directivas asociadas a la ejecución de un enfoque de liderazgo instruccional.

Se realizó un análisis de varianza 4x10 (Cargo x Función) para evaluar diferencias con respecto a la implementación de las actividades asociadas al desarrollo de liderazgo instruccional considerando el cargo en el establecimiento y las diez funciones directivas definidas en el marco conceptual de éste enfoque de liderazgo. Se determinó que existen diferencias significativas en la percepción de la implementación de actividades asociadas al liderazgo instruccional considerando los cargos que se desempeñan en el establecimiento educativo, $F(3,4767)=84.557, p<.001, \eta_{\mathrm{p}}{ }^{2}=.051$. Un análisis post hoc, de tipo Bonferroni reveló que no existen diferencias en la implementación de actividades de liderazgo instruccional considerando la autopercepción de los miembros de equipo directivo (Director, Jefe UTP y Coordinadores, $p>$.05), sin embargo, la percepción de los docentes con respecto al desarrollo de actividades asociadas al liderazgo instruccional por parte del equipo directivo fue significativamente menor, $p<.001$. Igualmente se establecieron diferencias significativas al comparar el desarrollo de actividades de liderazgo instruccional entre las diez funciones directivas comprendidas por el PIMRS, $F(9,4767)=2.319, p=.003, \eta_{p}{ }^{2}=.005$. El análisis post hoc, de tipo Bonferroni sólo reveló diferencias significativas entre la media de la implementación de actividades de liderazgo instruccional entre las funciones: Establece las metas de la escuela y Monitorea el progreso académico de la instrucción, $p<.024$. Las comparaciones entre todas las otras funciones no arrojaron diferencias entre ellas, $p>$.05. Finalmente, no se estableció una interacción significativa entre el cargo en el establecimiento y las funciones directivas del instrumento sobre la base del desarrollo de actividades de liderazgo instruccional $F(27,4767)=1.350, p=.080, \eta_{p}^{2}=.006$. 
Finalmente, al observar los promedios que reflejan los datos agrupados para los participantes del equipo de gestión (Directores, Jefes UTP y Coordinadores) y los docentes de los establecimientos educativos participantes es posible observar donde se encuentran las mayores diferencias entre la autopercepción de los integrantes de los equipos de gestión y la percepción de los docentes con respecto a la ejecución de funciones asociadas al liderazgo instruccional (figura 1). Las mayores brechas representadas por la línea continua en la gráfica se observan consistentemente las funciones que comprenden la tercera dimensión referida a fomentar un clima adecuado para el aprendizaje escolar. Por otro parte las menores brechas se aprecian en la ejecución de las funciones que comprenden la primera dimensión del modelo de liderazgo instruccional referido a definir la misión de la escuela.

\section{Discusión y conclusiones}

En esta investigación se analizan las competencias de liderazgo instruccional utlizando la Escala de Percepción de la Gestión Instruccional de Directores (PIMRS). Los valores de consistencia interna para las tres dimensiones y diez funciones del instrumento son equivalentes a los valores informados en estudios de validación del instrumento (Hallinger \& Wang, 2015; Hallinger et al., 2013) y datos tomados para una población similar a la de este estudio en Chile (Fromm et al., 2017)Rasch analysis. Los resultados encontrados en esta investigación confirman que el PIMRS es una herramienta que logra identificar habilidades de liderazgo instruccional reconocibles en diversos contextos educativos. Considerando los valores de consistencia interna de acuerdo a la función desempeñada en los establecimientos educativos, llama la atención que los Jefes UTP tienden a presentar valores de a de Cronbach menores con respecto a los otros cargos considerados en el estudio. Es posible que esto refleje diferentes énfasis en la atribución de responsabilidades respecto a actividades relacionadas al liderazgo instruccional en los distintos centros educativos.

En este estudio se compara la autovaloración asignada por los miembros del equipo de gestión y la valoración de los docentes con respecto a las dimensiones y funciones asociadas a la implementación de un estilo de liderazgo instruccional considerando el cargo ocupado en el centro educativo. No se econtraron diferencias en los niveles de valoración en función de cada dimensión definida en el PIMRS. Al realizar el mismo análisis por función, se estableció que la actividad con mayores índices de valoración fue Establece las metas de la escuela y la con menor valoración fue Monitorea el progreso académico de la instrucción. Las otras ocho funciones no se diferencian entre ellas con respecto a los niveles de valoración atribuida por los participantes del estudio. Por otro lado, el análisis por cargo determinó que la valoración de los docentes es significativamente menor a la valoración atribuida por los miembros del equipo de gestión, la que no se diferenció entre ellos. La existencia de diferencias en la percepción es normal que ocurra, lo interesante de constatar al desarrollar este tipo de perfiles, es que ofrece una oportunidad para comparar la autopercepción del director y equipo de gestión con respecto a las de los docentes (Hallinger, 2012). En este sentido, las menores brechas se observan en las funciones asociadas a la Dimensión 1 del PIMRS (Define la misión de la escuela) y las mayores brechas detectadas se asocian a las funciones incluidas en la Dimensión 3 del 
PIMRS (Fomenta un adecuado clima de aprendizaje escolar) donde se destacan las diferencias entre las funciones: Provee incentivos para los profesores y Provee incentivos para el aprendizaje (figura 1). Estos datos son consistentes a lo informado en una población similar a este estudio donde comparativamente los docentes presentan una alta valoración a la Dimensión 1 y una menor valoración a las funciones establecidas en la Dimensión 3, especificamente Provee incentivos para los profesores (Fromm et al., 2017)Rasch analysis, lo que tambien ocurre para los docentes que participan en este estudio.

La autopercepción con respecto al desarrollo de las funciones asociadas a la implementación de las funciones vinculadas al desarrollo de liderazgo instruccional por parte de los diferentes miembros de los equipos de gestión entregan interesantes elementos para el análisis. Uno de los desafíos de los directores escolares en el contexto chileno, es poner un mayor énfasis en actividades vinculadas al liderazgo pedagógico. En este sentido, con respecto a las funciones definidas en el PIMRS, se observa una menor valoración a las funciones asociadas a la Dimensión 2: Administra el plan académico de la escuela, específicamente a las tareas de Gestiona el currículo académico y Monitorea el progreso académico de la instrucción. Leiva, Montecinos, Ahumada, Campos, \& Guerra (2017), encuentran que la supervisión de la instrucción es una tarea difícil de lograr en directores nóveles lo que reconocen una alta demanda de labores administrativas. Por otro lado, los Jefes UTP asumen gran parte de las funciones asociadas a la toma de decisiones técnico-pedagógicas (Rodríguez Molina \& Gairín Sallán, 2017), lo que se ve reflejado en las más altas valoraciones en comparación a los directores y los otros miembros del equipo de gestión (tablas 5 y 6). La figura del Jefe UTP como un referente que asume gran parte de las responsabilidades instruccionales en la escuela no es común en otros sistemas educativos y establece desafíos con respecto a cómo los directores y Jefes UTP trabajan en conjunto para resolver los asuntos pedagógicos de la escuela que lideran (Flessa, 2014; Núñez, Weinsten \& Muñoz, 2010). En este sentido, es necesario avanzar hacia una estructura organizacional que permita lograr una visión compartida entre director y jefe de UTP acerca de aprendizajes de calidad y prácticas pedagógicas (Quiroga \& Aravena, 2017).

En términos generales las diferencias en la valoración atribuida a las funciones asociadas a la implementación de actividades de liderazgo instruccional entre los diferentes miembros de los equipos de gestión y la particularidad del rol del jefe de UTP obliga a remirar la relevancia del liderazgo directivo y el desarrollo de funciones pedagógicas en sintonía con el proyecto educativo de cada establecimiento. Un liderazgo pedagógico, implementado tanto por el director como por los miembros del equipo de gestión, especialmente por el jefe de UTP, genera un ambiente de trabajo orientado a metas comunes que, igual como se observa en las funciones menos presentes según los docentes, ayuda a estimular el trabajo profesional y su desarrollo como lo ha comentado la Organización para la Cooperación y el Desarrollo Económicos (OCDE, 2014) tras el último estudio sobre la enseñanza y el aprendizaje.

\section{Limitaciones y futuras investigaciones}

Si bien en la investigación participaron miembros de equipos docentes de 17 establecimientos, no se logró obtener la respuesta del PIMRS de todos los directores 
principalmente por estar ausentes al momento de la toma de mediciones. Notamos que para obtener una adecuada respuesta del instrumento, se recomienda que el mismo sea respondido de manera presencial explicando claramente los principales alcances de los datos recogidos. Esto limita de alguna manera obtener una mejor representación de establecimientos alejados de zonas urbanas.

Futuras investigaciones pueden estar dirigidas a lograr una mejor comprensión de las prácticas de liderazgo instruccional de los equipos directivos que demuestran fortalezas en las funciones identificadas en el PIMRS con respecto a sus pares. A través de una aproximación metodológica cualitativa sería posible lograr registrar en profundidad las prácticas asociadas a una destacada ejecución de liderazgo instruccional en el contexto del sistema de educación chileno, los cargos y las funciones atribuidas.

\section{Agradecimientos y Financiamiento}

Los autores agradecen a los directores y todo el equipo docente de los establecimientos educacionales de la región del Biobío que generosamente accedieron a participar y respondieron el instrumento, posibilitando el desarrollo de este estudio.

Esta investigación fue financiada a través de un proyecto de la Dirección de Investigación de la Universidad Católica de la Santísima Concepción, DIN 18/2016.

\section{Referencias}

Bush, T. (2016). Mejora escolar y modelos de liderazgo: Hacia la comprensión de un liderazgo efectivo. En J. Weinstein (Ed.), Liderazgo Educativo en la escuela. Nueve miradas (pp. 19-38). Santiago de Chile, Chile: Ediciones Diego Portales.

Dufour, R., \& Marzano, R. J. (2009). High-Leverage strategies for principal leadership. Educational Leadership, 66(5), 62-68.

Field, A. (2013). Discovering statistics using SPSS. SAGE Publications (4th ed.). London, UK: Sage Publications. doi: https://doi.org/10.1111/j.1365-2648.2007.04270_1.x

Flessa, J. (2014). Learning from school leadership in Chile. Canadian and International Education / Education Canadienne et Internationale, 43(1), 1-15. Recuperado de https:// ir.lib.uwo.ca/cie-eci/vol43/iss1/2

Flessa, J., Bramwell, D., Fernández, M., \& Weinstein, J. (2018). School leadership in Latin America 2000-2016. Educational Management Administration and Leadership, 46(2), 182-206. doi: https://doi.org/10.1177/1741143217717277

Fromm, G., Hallinger, P., Volante, P., \& Wang, W. C. (2017). Validating a spanish version of the PIMRS: Application in national and cross-national research on instructional leadership. Educational Management Administration and Leadership, 45(3), 419-444. doi: https://doi.org/10.1177/1741143215617948

Fullan, M. (2014). The principal: Three keys to maximizing impact. San Francisco, CA, EE.UU.: Jossey-Bass.

Hallinger, P. (2011). Leadership for learning: lessons from 40 years of empirical research. Journal of Educational Administration, 49(2), 125-142. doi: https://doi. org/10.1108/09578231111116699 
Hallinger, P. (2012). A data-driven approach to assess and develop instructional leadership with the PIMRS. En J. Shen (Ed.), Tools for Improving Principals' Work (pp. 47-69). New York, NY, EE.UU.: Peter Lang.

Hallinger, P., \& Murphy, J. (1985). Assessing the instructional management behavior of principals. The Elementary School Journal, 86(2), 217. https://doi.org/10.1086/461445

Hallinger, P., Wang, W.-C., \& Chen, C.-W. (2013). Assessing the measurement properties of the principal instructional management rating scale: A meta-analysis of reliability studies. Educational Administration Quarterly, 49(2), 272-309. doi: https:// doi.org/10.1177/0013161X12468149

Hallinger, P., \& Wang, W. C. (2015). Assessing instructional leadership with the principal instructional management rating scale. London: Springer International. doi: https:// doi.org/10.1007/978-3-319-15533-3

Hargreaves, A., \& Fullan, M. (2014). Capital profesional. Transformar la enseñanza en cada escuela. Madrid: Ediciones Morata.

Kouzes, J. M., \& Posner, B. Z. (2012). The leadership challenge: How to make extraordinary things happen in organizations (5th ed.). San Francisco, CA, EE.UU.: Jossey-Bass.

Leithwood, K., Day, C., Sammons, P., Harris, A., \& Hopkins, D. (2006). Successful school leadership what it is and how it influences pupil learning. National College for School Leadership, 132.

Leiva, M. V., Montecinos, C., Ahumada, L., Campos, F., \& Guerra, S. (2017). Novice principals' instructional management practices in high poverty, low performing schools in Chile. Procedia - Social and Behavioral Sciences, 237, 3-9.

Mansilla, J., \& Beltrán, J. (2016). Racionalidad instrumental y prácticas de gestión en jefes técnicos de liceos de la Araucanía Instrumental rationality and management practices in technical heads of schools of Araucanía. Revista de Investigación Educativa, 34(1), 151-165. doi: https://dx.doi.org/10.6018/rie.34.1.218041

Marzano, R. J., Waters, T., \& McNulty, B. A. (2005). School leadership that works: From research to results (1st ed.). Alexandria,VA, EE.UU.: ASCD.

Maureira, Ó. (2018). Prácticas del liderazgo educativo: Una mirada evolutiva e ilustrativa a partir de sus principales marcos, dimensiones e indicadores más representativos. Revista Educación, 42(1), 1-19. doi: https://dx.doi.org/10.15517/REVEDU.V42I1.22115

Ministerio de Educación de Chile [Mineduc] (2015). Marco para la buena dirección y el liderazgo escolar. Santiago de Chile, Chile. Recuperado de http://liderazgoescolar. mineduc.cl/wp-content/uploads/sites/55/2016/04/MBDLE_2015.pdf

Ley № 20.529, de 11 de agosto, por el que se crea el Sistema de Aseguramiento de la Calidad de la educación parvularia, básica y media y su fiscalización. Ministerio de Educación de Chile, 27 de agosto de 2011.

Ley № 21.040, de 16 de noviembre, por el que se crea el Sistema de Educación Pública. Ministerio de Educación de Chile, 24 de noviembre de 2017.

Murillo, J. (2012). La dirección escolar en Chile: Una visión en el contexto de América Latina. En J. Weinstein y G. Muñoz (Eds.), ¿Que sabemos sobre los directores en Chile? (pp. 19-40). Santiago: Salesianos Impresores S.A.

Núñez, I., Weinstein, J., \& Muñoz, G. (2010). ¿Posición olvidada? una mirada desde la normativa a la historia de la dirección escolar en Chile. Psicoperspectivas, 9(2), 53-81. doi: https://dx.doi.org/10.5027/psicoperspectivas-Vol9-Issue2-fulltext-117 
Organizacion para la Cooperación y el Desarrollo Economics [OCDE] (2014). Talis 2013 results: An international perspective on teaching and learning. París, Francia: Autor. doi: https://doi.org/10.1787/9789264216075-en

Oficina Regional de Educación para América Latina y el Caribe de la Organización de las Naciones Unidas para la Educación [OREALC-UNESCO] (2015). Informe Anual 2014. Santiago de Chile, Chile: UNESCO.

Quiroga, M., \& Aravena, F. (2017). Jefes de UTP nóveles en Chile: desafíos del liderazgo pedagógico. Perspectiva Educacional, 56(3), 76-97. Recuperado de http://www. perspectivaeducacional.cl/index.php/peducacional/article/view/506

Robinson, V. M. J., Lloyd, C. A., \& Rowe, K. J. (2008). The impact of leadership on student outcomes: an analysis of the differential effects of leadership types. Educational Administration Quarterly, 44(5), 635-674. doi: https://doi.org/10.1177/0013161X08321509

Rodríguez Molina, G. A., \& Gairín Sallán, J. (2017). Influencia de las prácticas de liderazgo pedagógico en las prácticas pedagógicas docentes: caso en Chile de las Unidades Técnicas Pedagógicas. International Journal of Educational Leadership and Management, 5(1), 6. doi: https://doi.org/10.17583/ijelm.2017.2469

Sepúlveda, F., \& Aparicio, C. (2017). El desafío de los directores de escuelas chilenas : Liderando a partir de un enfoque instruccional hacia un enfoque distribuido. Revista Gestion de la Educación, 7(2), 1-19. Recuperado de https://revistas.ucr.ac.cr/index. php/gestedu/article/view/30599

Vaillant, D., \& Zidán, E. R. (2016). Prácticas de liderazgo para el aprendizaje en América Latina: un análisis a partir de PISA 2012. Ensaio: Avaliação E Políticas Públicas Em Educação, 24(91), 253-274. doi: https://doi.org/10.1590/S0104-40362016000200001

Weinstein, J., \& Muñoz, G. (Eds.) (2012). ¿Qué sabemos sobre los directores en Chile? Santiago de Chile, Chile: Salesianos Impresores S.A.

Fecha de recepción: 2 de mayo de 2018.

Fecha de revisión: 18 de mayo de 2018.

Fecha de aceptación: 19 de diciembre de 2018. 
